\title{
Aktinische Keratosen - erfolgreicher Einsatz von Imiquimod
}

\section{R. Stadler}

\author{
Actinic Keratoses - Successful Treatment with Imiquimod
}

\section{Zusammenfassung}

In der vorliegenden Kasuistik wird über den erfolgreichen Einsatz von Imiquimod bei einem 80-jährigen Patienten mit ausgeprägten aktinischen Keratosen berichtet. Nach dreimal wöchentlicher Anwendung für $10^{1} / 2$ Wochen von Imiquimod über Nacht konnte eine komplette Abheilung beobachtet werden.

\section{Abstract}

In this case-presentation we report on a successful treatment with Imiquimod in an 80-year-old patient with extensive actinic keratoses. After applying Imiquimod three times a week at night for $10^{1} / 2$ weeks a complete resolution of the actinic keratoses could be observed.
Aktinische Keratosen stellen die häufigste Präkanzerose dar. Sie treten meistens multipel etwa ab dem 50. Lebensjahr auf chronisch UV-geschädigter Haut auf und in Abhängigkeit vom Hauttyp und langjähriger UV-Belastung im Laufe des Lebens.

Die Therapie der aktinischen Keratosen basiert bis heute im Wesentlichen auf dem Einsatz von flüssigem Stickstoff, Kryotherapie, unterschiedlichen Ablationsverfahren mit $\mathrm{CO}_{2}$ - bzw. Erbium:YAG-Laser als auch auf der Anwendung von Antimetaboliten wie der 5-Fluouracil-Creme. In dieser Farbbildkasuistik wird beispielhaft über die erfolgreiche Therapie mit 5\% ImiquimodCreme berichtet.

\section{Fallbeschreibung}

Ein 80 Jahre alter Mann kam zur Behandlung mit multiplen, flächig flach papulösen, erythematösen und krustösen, zum Teil nässenden Effloreszenzen im Bereich der Wange, Stirn und Kopfhaut (Abb. 1 a u. b).

Diese Hautveränderungen waren innerhalb der letzten fünf Jahre aufgetreten und wiederholt mit einer oberflächlichen $\mathrm{CO}_{2}$-Lasertherapie behandelt worden. Nach kurzfristiger Abheilung sei es jedoch schnell zum erneuten Rezidiv und Fortschreiten der bestehenden Hauterscheinungen gekommen.

Die Ganzkörperuntersuchung des Patienten war ohne pathologischen Befund. Aus einer erythematösen papulösen Effloreszenz wurde eine Gewebeprobe entnommen, die histologisch einer aktinischen Keratose entsprach.

Daraufhin wurde eine Lokaltherapie mit Imiquimod-Creme, dreimal wöchentlich flächig über Nacht auf Stirn und Kopfpartie aufgetragen, eingeleitet. Unter der lokalen Anwendung deutliche Demarkierung der behandelten erythematösen, makulopapulösen Effloreszenzen unter subjektiv brennenden Symptomen. Diese sind nach Angaben des Patienten gut zu tolerieren. Nach 10,5 Wochen klinisch komplette Abheilung der aktinischen Keratosen (Abb. 2 a u. b).

\section{Diskussion}

Topische Immuntherapeutika mit immunstimulierenden Wirkungen wurden in jüngster Zeit erfolgreich in der Therapie entzündlicher, infektiöser und präkanzeröser sowie kanzeröser Hauterkrankungen eingesetzt $[1,3,4]$. Imiquimod gehört zur Fa- 


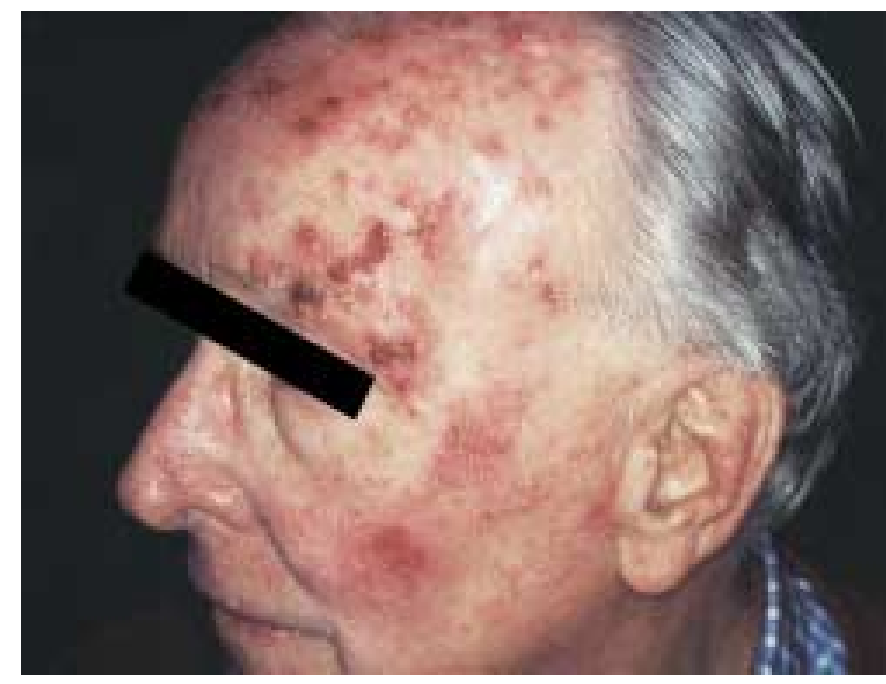

Abb. 1 a Aktinische Keratose vor Therapie.

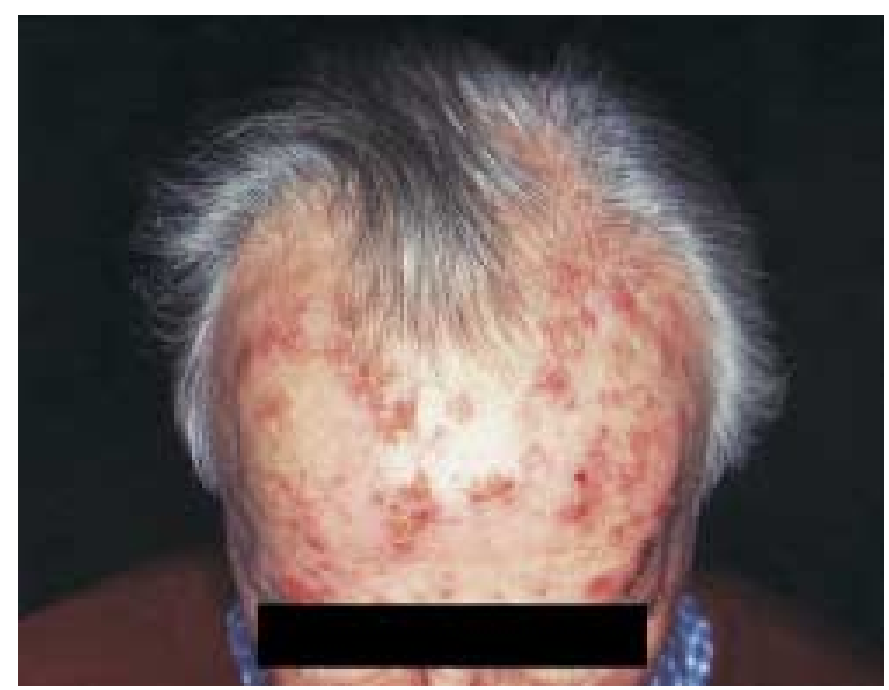

Abb. 1 b Aktinische Keratose vor Therapie.

milie der Imidazolquinoline, die antivirale und antitumorale Eigenschaften in Tiermodellen gezeigt haben. Diese Immunmechanismen werden mediiert durch Stimulation sowohl der unspezifischen als auch der spezifischen zellmediierten Immunität. Es werden durch Imiquimod zahlreiche Zytokine induziert, zu denen Interferon $\alpha$, der Tumornekrosefaktor $\alpha$, Interleukin-1-Rezeptor Antagonist, Interleukin-12 und auch Interferon $\gamma$ als TH1-Immunantwort zählen. Darüber hinaus wird eine Reihe von weiteren antiviralen Proteinen induziert [2].

In einer Phase-II-Studie konnte durch die Behandlung von aktinischen Keratosen mit 5\% Imiquimod-Creme eine histologische Abheilung von bis zu $87 \%$ erzielt werden [4].

In der hier vorgestellten Farbbildkasuistik führte die Therapie mit 5\% Imiquimod-Creme über einen Anwendungszeitraum von 6 Wochen dreimal wöchentlich appliziert zu einer klinisch kompletten Abheilung sämtlicher aktinischer Keratosen nach 10,5 Wochen. Die Abheilung erfolgte über eine klinisch deutlich sichtbare inflammatorische Reaktion. Diese sollte dem Patienten

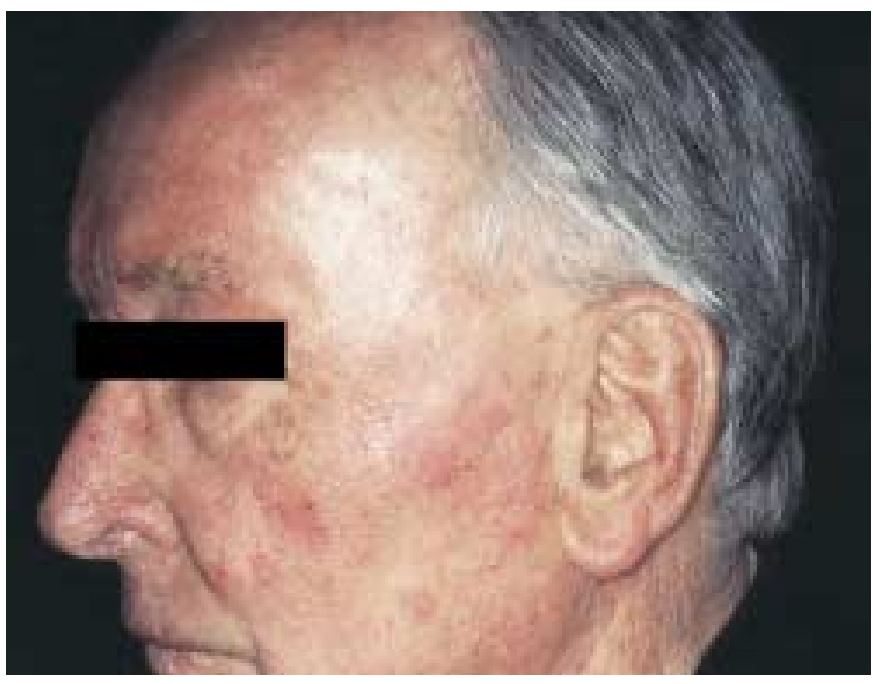

Abb. 2a Nach 10,5 Wochen Therapie mit Imiquimod.

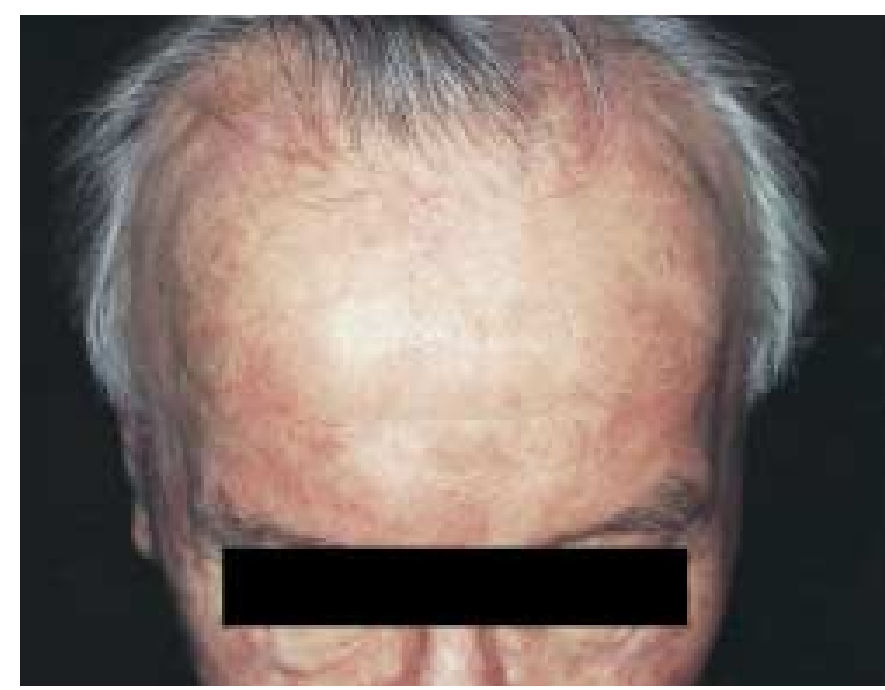

Abb. 2b Nach 10,5 Wochen Therapie mit Imiquimod.

vor Therapieeinleitung erläutert werden. Sie ist als Ausdruck der Zytokininduktion und möglicherweise für das therapeutische Ansprechen erforderlich zu werten.

Über ähnliche Erfolge wurde in jüngerer Zeit für das oberflächliche Basalzellkarzinom berichtet [1]. Zu dieser Indikation werden zur Zeit nationale und internationale Multizenterstudien durchgeführt. Im Vergleich zu bisher zur Verfügung stehenden Therapieverfahren bei ausgedehnten aktinischen Keratosen stellt Imiquimod eine patientenfreundliche und sehr elegante Therapiemöglichkeit dar, die einer kontrollierten Evaluierung in entsprechenden Studienprojekten bedarf. 


\section{Literatur}

${ }^{1}$ Beutner KR, Geisse JK, Helman D, Fox TL, Ginkel A, Owens ML. Therapeutic response of basal cell carcinoma to the immune response modifier imiquimod 5\% cream. J Am Acad Dermatol 1999; 41: $1002-1007$

${ }^{2}$ Hengge UR, Stark R. Topical imiquimod to treat intraepidermal carcinoma. Arch Dermatol 2001; 137: 709-711
${ }^{3}$ Marks R, Gebauer K, Shumack S, Amies M, Breyden J, Fox TL et al. Imiquimod $5 \%$ cream in the treatment of superficial basal cell carcinoma: results of a multicenter 6-week dose-response trial. J Am Acad Dermatol 2001; 44: 807-813

${ }^{4}$ Stockfleth E, Meyer T, Benninghoff B, Christophers E. Successful treatment of actinic keratosis with imiquimod cream $5 \%$ : a report of six cases. Br J Dermatol 2001; 144: 1050-1953

\section{Buchbesprechung}

\section{Dermatologie, Allergologie, Umweltmedizin (Springer Enzyklopädie) \\ Altmeyer P., M. Bacharach-Buhles \\ Springer 2002, 1347 Abb., 566 Tab., 1852 S. Geb., € 199,00, \\ ISBN 3-540-41361-8}

Bei der vorliegenden Enzyklopädie schaffen P. Altmeyer und M. Bacharach-Buhles ein umfassendes, durch die lexikalische Struktur äußerst übersichtliches Nachschlagewerk der Dermatologie, Allergologie und Umweltmedizin sowie Venerologie, Proktologie und ästhetisch korrektiven Verfahren.

Dank der alphabetischen Reihenfolge sind Einträge, insgesamt über 13000 Stichworte, rasch auffindbar. Nach jeweils kurzer und prägnanter Definition folgen Absätze zu Krankheitsmanifestation, Lokalisation, Klinik und Differentialdiagnose. Unterstützend erleichtern und illustrieren ca. 1500 klinische und histologische Abbildungen die Diagnosefindung. Interessant ist die historische Kommentierung mit Nennung von Erstbeschreibern von relevanten dermatologischen Krankheitsbildern. Darüber hinaus bietet das Werk dem historisch Interessierten die Möglichkeit, knappe biographische Daten prominenter Dermatologen nachzuschlagen.
Auch die Therapieoptionen werden zum jeweiligen Krankheitsbild ausführlich aufgeführt und ggf. auch kritisch kommentiert, inklusive naturheilkundlicher Therapieansätze. Unterschieden wird jeweils interne und externe Therapie sowie operative Möglichkeiten. In einem gesonderten Anhang finden sich darüber hinaus insgesamt 328 Rezepturen.

Insgesamt ist es den Autoren gelungen, in kurzer, präziser aber umfassender Form ein Nachschlagewerk für Dermatologie, Allergologie und Umweltmedizin zu schaffen. Es ermöglicht nicht nur dem Dermatologen sondern auch Ärzten aus anderen Fachbereichen, sich einen schnellen Überblick über das Fachgebiet zu verschaffen und bei weitergehendem Interesse sich mittels der angegebenen Literaturstellen tiefergehend zu informieren.

Dank der beiliegenden CD-Rom mit zoombaren Abbildungen und Möglichkeiten der Volltextrecherche steht auch ein modernes Medium für interaktives Arbeiten und Lernen z.B. für den Arzt in der Weiterbildung zur Verfügung.

U. Gleichmann, Minden 